\title{
Comparação do desenvolvimento motor de lactentes de mães adolescentes e adultas
}

\author{
Motor development comparison between infants of adolescent and adult mothers
}

\author{
Naiane Sartori ${ }^{1}$, Raquel Saccani ${ }^{2}$, Nadia C. Valentini ${ }^{3}$
}

Estudo desenvolvido no Centro de Ciências da Saúde da UCS - Universidade de Caxias do Sul, Caxias do Sul, RS, Brasil

1 Fisioterapeuta

2 Profa. Ms. do Curso de Fisioterapia da UCS

3 Profa. Dra. do Curso da Escola Superior de Educação Física da Universidade Federal do Rio Grande do Sul, Porto Alegre, RS

\section{ENDERECO PARA} CORRESPONDÊNCIA:

Raquel Saccani Av. Loureiro da Silva 1788 ap.209 Cidade Baixa 90050-240 Porto Alegre RS e-mail: raquelsaccani@yahoo.com.br

\section{APRESENTAÇÃO}

abr. 2010

ACEITO PARA PUBLICAÇÃO jun. 2010
Resumo: Este estudo objetivou caracterizar o desempenho motor de bebês de 0 a 16 meses, comparando o de bebês nascidos de mães adolescentes ao de bebês de mães adultas. Participaram 80 bebês, sendo 40 de mães adolescentes e 40 de mães adultas. Os instrumentos avaliativos foram a escala motora infantil de Alberta AIMS (Alberta infant motor scale) e questionário para identificar as características biológicas. Os dados foram analisados estatisticamente. Os resultados da AIMS mostram pior desempenho motor dos bebês de mães adolescentes $(p=0,002 ; p=0,001)$, além de suas inadequadas aquisições motoras nas posturas prono e em pé. Não foi encontrada associação significativa entre a maioria dos fatores biológicos analisados e o desenvolvimento motor dos bebês. Os resultados sugerem que a idade materna pode ser considerada fator de risco para atraso motor em crianças, porém ressalta-se a interação de diferentes fatores, aqui não controlados, influenciando as aquisições motoras de crianças, tais como as demandas da tarefa e as condições do ambiente. Descritores: Atividade motora; Desenvolvimento infantil; Fatores de risco; Gravidez na adolescência; Lactente

Abstract: The purpose of the study was to compare motor development of infants aged 0 to 16 months born of teenage mothers to those of adult mothers. Eighty babies were assessed, 40 of which the children of teenage mothers, and 40 of adult mothers. Instruments used were the Alberta infant motor scale (AIMS) and a questionnaire to collect biological information. Data were statistically analysed. Results show teenage mothers' infants to have delayed motor development as compared to adult mothers' ones $(p=0.002 ; p=0.001)$, as well as their poor motor acquisitions in prone and standing positions. No association was found between infants' motor development and most of the biological factors analysed. Results hence suggest that early maternal age may be considered a risk factor to motor delay in infants, but attention should be paid to other factors such as task requirements and environmental conditions (here not controlled) that may influence the pace of motor acquisition in infants.

Key words: Child development; Infant; Motor activity; Pregnancy in adolescence; Risk factors 


\section{INTRODUÇÃO}

Considerando a repercussão dos atrasos motores no desenvolvimento do indivíduo, é de extrema importância identificar as crianças expostas a maiores riscos, para minimizar os resultados daí decorrentes 1,2. São considerados fatores de risco diversas condições que podem ocasionar atrasos no desenvolvimento motor, social e cognitivo da criança ${ }^{2}$. A gestação na adolescência é apontada como um possível fator de risco para atrasos no crescimento 3 e nas aquisições motoras da criança ${ }^{4}$, visto que tem sido relacionada ao vínculo mãe-bebê deficiente, à negligência materna, falta de cuidados adequados e desinteresse $\mathrm{e}^{5-8}$, além da prematuridade 7,9-11 e do baixo peso ao nascer 4,7,9-13, entre outros fatores, o que pode resultar em desvantagens no desenvolvimento dessas crianças quando comparadas às nascidas de mães adultas. A gravidez na adolescência também tem sido associada ao aumento de intercorrências hospitalares 11 , bem como ao maior índice de morbidade e mortalidade infantil e neonatal5,9. Destacam-se ainda alguns comportamentos de risco relacionados à adolescência, como depressão, baixa auto-estima11, uso de drogas ilícitas, consumo de álcool6-8 e tentativas de aborto ${ }^{8}$.

Em serviços de saúde coletiva são comuns gestantes adolescentes 7,8,10,13,14, bem como de bebês com alterações no desenvolvimento motor ${ }^{2}$. Nesse sentido, é necessário não apenas identificar precocemente atrasos motores, mas também detectar possíveis fatores de risco que podem influenciar negativamente o desenvolvimento. No entanto, são escassas as pesquisas sobre a gravidez na adolescência que focalizem a idade materna como fator de risco para atraso no desenvolvimento motor de bebês.

Estudos reforçam o entendimento de que o desenvolvimento adequado dependerá da qualidade das experiências vividas pela criança e dos vínculos por ela criados 15-17. Estabelece-se pois a necessidade da identificação precoce de crianças expostas a fatores de risco, assim como a avaliação de seu desenvolvimento, com o objetivo de minimizar possíveis atrasos motores 2,18. Programas de intervenção motora precoce podem potencializar o processo de desenvolvimento e intensificar as oportunidades de aquisições motoras 17 , pois acredita-se que a criança considerada vulnerável pode suplantar a desvantagem, desde que o ambiente forneça experiências compensatórias 19 . Assim, o reconhecimento da influência dos diferentes fatores sobre o desenvolvimento infantil pode subsidiar propostas de abordagem precoce, para minimizar eventuais efeitos comportamentais indesejáveis².

Portanto, essa pesquisa teve como objetivos caracterizar e comparar o desempenho motor de bebês nascidos de mães adolescentes com o de bebês de mães adultas, bem como verificar possíveis associações entre suas características biológicas e o desempenho motor.

\section{METODOLOGIA}

Este foi um estudo descritivo, de abordagem transversal20, aprovado pelo Comitê de Ética em Pesquisa da Fundação Universidade Caxias do Sul e pela Prefeitura de Caxias do Sul. A amostra foi composta de 80 bebês com idade entre 0 e 16 meses, residentes numa comunidade de Caxias do Sul, RS, cadastradas em uma unidade básica de saúde (UBS) da cidade. As condições socioeconômicas da população da região eram razoáveis: as moradias dos participantes tinham saneamento básico, três a cinco cômodos e a renda familiar variou de 2 a 4 salários mínimos, sendo a média de 2,7 (+0,35). Considerou-se que os participantes apresentavam equivalência quanto a aspectos socioeconômicos pelo fato de residirem na mesma comunidade.

A amostra foi dividida em dois grupos: o grupo A, composto por 40 bebês de mães adolescentes (12 a 19 anos), e o grupo B, composto por 40 bebês com idade e sexo pareados ao grupo A, de mães adultas (acima de 20 anos). A amostra foi estabelecida com base na população da comunidade, mediante pesquisa prévia em prontuários de participantes do projeto "De Olho no Risco", da Prefeitura Municipal de Caxias do Sul, que mapeia todas as crianças expostas a riscos para atraso, dentre os quais se inclui a gestação na adolescência (até 19 anos). Foram incluídos todos os bebês nascidos de mães adolescentes com idade entre 0 e 18 meses; foram excluídas crianças prematuras, com muito baixo peso ao nascer $(<1500$ g), com comprometimento osteoarticular ou neurológico e aquelas que já participavam de projetos interventivos.
Os bebês tinham entre 0 e 16 meses, em média 6,65 $\pm 4,32$ meses. Quanto à distribuição por faixa etária, 24 bebês (30\%) estavam no 1o trimestre de vida, 16 bebês (20 \%) no 2o, $22(27,5 \%)$ no 3o, $10(12,5 \%)$ no 4 o e 8 bebês (10\%) no 5 o trimestre de vida. Quanto ao sexo, 46 $(57,5 \%)$ eram do sexo feminino.

Os instrumentos de avaliação utilizados foram a escala motora infantil de Alberta AIMS (Alberta infant motor scale) e um questionário estruturado sobre as condições biológicas do bebê, respondido pela responsável da criança. A AIMS permite avaliar o desempenho motor de recém nascidos até os 18 meses de idade. Propõe a observação de 58 itens, agrupados em 4 subescalas segundo as posições: 21 itens em prono, 9 em supino, 12 sentado e 16 em pé. $\mathrm{O}$ instrumento pressupõe mínima manipulação, analisando-se, para cada postura, descarga de peso, alinhamento postural e movimentos antigravitacionais, observando os padrões de postura do bebê de acordo com a idade 21 .

Cada item é avaliado segundo tenha sido observado (escore 1) ou não-observado (escore 0). Os escores em cada subescala são somados; a soma dos quatro subtotais é convertida para percentil de desempenho motor, estabelecido com base na amostra normativa do teste. A criança foi classificada como: desempenho motor normal, se acima de $25 \%$ da curva percentílica; desempenho motor suspeito, entre $25 \%$ e $5 \%$ da curva; e desempenho motor atrasado, abaixo de 5\% 21 .

O questionário referente às condições biológicas do bebê pesquisou os seguintes aspectos: data de nascimento; semanas de gestação; índice de Apgar; peso ao nascer; comprimento ao nascer; perímetro cefálico; período (dias) de internação em unidade de terapia intensiva (UTI) ao nascer; e tipo de parto.

A coleta de dados iniciou-se com o levantamento do número de crianças cadastradas na UBS, verificando-se quantas e quais correspondiam aos critérios de inclusão estabelecidos. Depois foram feitos contatos, sendo as crianças avaliadas em seus domicílios. Os responsáveis pelos bebês foram informados e esclarecidos da pesquisa, tendo assinado o termo de consentimento livre e esclarecido. Finalmente, foi preenchido o questionário referente às condições biológicas e realizada a avaliação do desempenho motor. 
A aplicação da AIMS foi feita por um mesmo avaliador nas residências. Todas as avaliações foram registradas em filmagem (câmera Kodak AF-3 Optical Aspheric Lens). Um avaliador independente avaliou o desempenho motor das crianças observando as filmagens; a confiabilidade entre os avaliadores foi de 0,81, o que está de acordo com o proposto pelas autoras do teste, que sugerem adequados os valores acima de $80 \% 21$. As filmagens foram usadas unicamente para fins científicos, sem exposição dos participantes, os quais tiveram sua identidade preservada.

Os dados coletados foram analisados pelo programa estatístico SPSS (v.14.0). Foi utilizada estatística descritiva com distribuição de freqüência simples e relativa, bem como as medidas de tendência central (média/mediana) e de variabilidade (desvio padrão). Para as associações da categorização do desenvolvimento motor das crianças entre os grupos e com as condições biológicas analisadas, foi utilizado o teste qui-quadrado de Pearson, com nível de significância de 5\%, e o eta2, onde foram considerados valores acima de 0,60 como indicativos de associação forte; valores entre 0,30 e 0,60, associação moderada; e valores abaixo de 0,3 , pobre. Para as comparações entre grupos de variáveis quantitativas (escore total, percentil, subescores), foi aplicado o teste de normalidade (Kolmogorov Smirnov) e, devido à distribuição não-paramétrica dos dados, foi adotado o teste U de Mann-Whitney, considerando-se nível de significância de 5\%22.

\section{RESULTADOS}

No que se refere às características biológicas gerais dos 80 bebês, observou-se que poucas crianças ficaram internadas em UTI (média de 0,46+1,68 dias); quanto ao tipo de parto, $76,3 \%(\mathrm{n}=61)$ das crianças nasceram de parto normal e 23,8\% ( $n=19)$, de cesárea. A descrição das demais características biológicas está disposta na Tabela 1 , onde se observa homogeneidade entre grupos $(p>0,05)$.

Quanto ao desempenho motor dos participantes, verificou-se que $11,3 \%(n=9)$ demonstraram desenvolvimento motor atrasado, 21,3\% ( $\mathrm{n}=17)$ foram classificados como desenvolvimento motor suspeito e $67,5 \%(n=54)$ apresentaram desenvolvimento motor adequado. Considerando
Tabela 1 Características biológicas (média \pm desvio padrão) dos bebês dos grupos A (de mães adolescentes, $n=40$ ) e B (de mães adultas, $n=40$ )

\begin{tabular}{lcccc}
\hline Característica & Total $(\mathrm{n}=80)$ & Grupo A & Grupo B & $p$ \\
\hline Idade gestacional (semanas) & $38,9 \pm 1,45$ & $39,0 \pm 1,32$ & $39,1 \pm 1,15$ & 0,66 \\
Peso ao nascer $(\mathrm{kg})$ & $3,23 \pm 0,65$ & $3,30 \pm 0,62$ & $3,16 \pm 0,68$ & 0,38 \\
Apgar (mediana) & 9,$0 ;$ & 9,0 & 9,0 & 0,10 \\
Comprimento ao nascer $(\mathrm{cm})$ & $49,1 \pm 3,30$ & $48,7 \pm 3,16$ & $49,5 \pm 3,44$ & 0,91 \\
Perímetro cefálico $(\mathrm{cm})$ & $35,0 \pm 3,20$ & $34,9 \pm 3,28$ & $35,1 \pm 3,16$ & 0,95 \\
\hline
\end{tabular}

$p$ da comparação entre os grupos

os grupos, os bebês de mães adolescentes (GA) apresentaram pior desempenho motor, comparados aos bebês de mães adultas, conforme o Gráfico 1.

Os escores brutos e nas subescalas da AIMS, assim como a classificação dos bebês dos dois grupos nas categorias da AIMS são apresentados nas Tabelas 2 e 3 . Na comparação entre os grupos, considerando a classificação, observou-se diferença significativa de desempenho $\left(\right.$ chi $^{2}=12,19$; $p=0,002$ ) entre os grupos: crianças do grupo A apresentaram maior freqüência de atraso, não observado no grupo B. Entretanto, considerando o escore total na AIMS, não foram observadas diferenças significativas entre os grupos, nas quatro posturas analisadas.

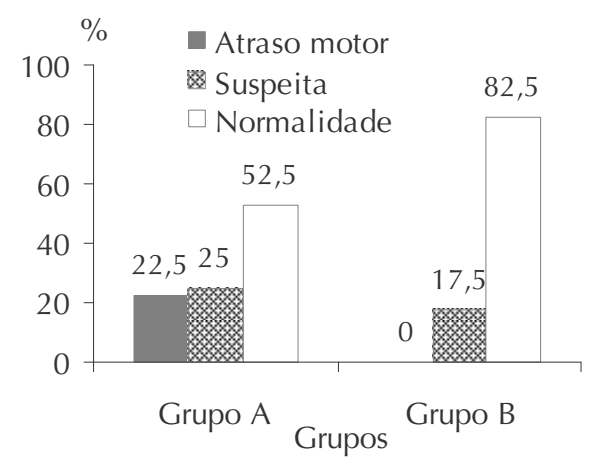

Gráfico 1 Distribuição (\%) da classificação obtida na AIMS pelos bebês dos grupos A (de mães adolescentes, $n=40$ ) e B (de mães adultas, $\mathrm{n}=40$ )

Tabela 2 Escores na AIMS (média \pm desvio padrão) e classificação do desempenho motor dos bebês dos grupos A (de mães adolescentes, $\mathrm{n}=40$ ) e $\mathrm{B}$ (de mães adultas, $\mathrm{n}=40$ )

\begin{tabular}{|c|c|c|c|c|c|c|c|}
\hline Desempenho motor & \multicolumn{2}{|c|}{ Total $(n=80)$} & \multicolumn{2}{|c|}{ Grupo A } & \multicolumn{2}{|c|}{ Grupo B } & $p$ \\
\hline Escore bruto & \multicolumn{2}{|c|}{$28,56 \pm 18,7$} & \multicolumn{2}{|c|}{$26,38 \pm 18,25$} & \multicolumn{2}{|c|}{$30,75 \pm 18,1$} & 0,25 \\
\hline Escore percentílico (\%) & \multicolumn{2}{|c|}{$42,4 \pm 27,2$} & \multicolumn{2}{|c|}{$32,5 \pm 27,6$} & \multicolumn{2}{|c|}{$52,5 \pm 23,0$} & 0,001 \\
\hline Classificação & $\mathrm{n}$ & $\%$ & $\mathrm{n}$ & $\%$ & $\mathrm{n}$ & $\%$ & \\
\hline Atrasado & 9 & 11,3 & 9 & 22,5 & 0 & 0,0 & 0,002 \\
\hline Suspeito & 17 & 21,3 & 10 & 25,0 & 7 & 17,5 & \\
\hline Normal & 54 & 67,5 & 21 & 52,5 & 33 & 82,5 & \\
\hline
\end{tabular}

AIMS = Escala motora infantil de Alberta; $p$ da comparação entre os grupos

Tabela 3 Escores (média \pm desvio padrão; mediana) nas subescalas da AIMS obtidos pelos bebês dos grupos A (de mães adolescentes, $n=40$ ) e B (de mães adultas, $\mathrm{n}=40$ )

\begin{tabular}{lcccc}
\hline Posturas da AIMS & Total $(\mathrm{n}=80)$ & Grupo A & Grupo B & $p$ \\
\hline Prono & $10,23 \pm 7,68 ; 9,5$ & $9,10 \pm 7,84 ; 5,0$ & $11,35 \pm 7,44 ; 11,0$ & 0,14 \\
Supino & $6,39 \pm 2,82 ; 8,0$ & $5,98 \pm 2,94 ; 5,5$ & $6,80 \pm 2,66 ; 8,0$ & 0,32 \\
Sentado & $6,48 \pm 4,67 ; 7,0$ & $5,85 \pm 4,54 ; 5,0$ & $7,10 \pm 4,77 ; 9,0$ & 0,25 \\
Em pé & $5,98 \pm 7,09 ; 3,5$ & $6,45 \pm 8,96 ; 3,5$ & $5,50 \pm 4,59 ; 3,5$ & 0,64 \\
\hline
\end{tabular}

AIMS = Escala motora infantil de Alberta; $p$ da comparação entre os grupos 
A análise de associação referente aos valores percentílicos revela que a variação destes está relacionada aos grupos de análise (eta $\left.{ }^{2}=0,57\right)$, ou seja, os percentis mais altos de desempenho foram observados no grupo B.

Ao se associar o desempenho motor dos bebês de forma geral com as características biológicas, não foi encontrada associação significativa com o comprimento ao nascer $\left(\mathrm{eta}^{2}=0,11\right)$; $\operatorname{sexo}\left(\mathrm{chi}^{2}=0,98\right.$; $p=0,61)$; Apgar no $5 \underline{\text { O }}$ minuto $\left(\mathrm{eta}^{2}=0,02\right)$; perímetro cefálico $\left(\mathrm{eta}^{2}=0,16\right)$; tempo de UTI ao nascer $\left(\right.$ eta $\left.^{2}=0,15\right)$ e tipo de parto $\left(\right.$ chi $\left.^{2}=0,50 ; p=0,77\right)$. Foi observada forte associação do desempenho motor apenas com peso ao nascer ( eta $^{2}=0,67$ ). Quando as associações foram pesquisadas intra-grupos, no grupo B não foi encontrada associação significativa entre o desempenho motor e as características biológicas; no grupo A, foi encontrada associação forte entre desempenho motor e peso ao nascer ( eta $^{2}=0,88$ ), sendo que os bebês com maior peso ao nascer obtiveram melhor desempenho motor.

\section{DISCUSSÃO}

No presente estudo, observou-se diferença significativa entre o desempenho dos bebês filhos de adolescentes e os nascidos de mães adultas, ressaltando a possibilidade de a idade materna ser um fator de risco para atraso no desenvolvimento. Estudos sugerem que as mães adolescentes estariam pouco preparadas para cuidar dos filhos, devido à dificuldade de adaptação à nova vida, o que poderia se refletir, até, em abandono futuro 2,11. Além disso, embora não controlados neste estudo, acredita-se que na maternidade adolescente incidam diferentes fatores considerados de risco, já que a literatura associa a pouca idade da mãe a estímulos inadequados à criança ${ }^{5-8}$, rejeição 8 , falta de cuidados pré e pós-natais $5,6,11,13$, uso de drogas, bebidas6-8 e à depressão 11 , que podem gerar impacto no desenvolvimento intelectual e motor do bebê. Apesar de não terem sido pesquisadas características das mães, os resultados do presente estudo parecem concordar com essa preocupação, uma vez que um quinto das crianças nascidas de mães adolescentes apresentaram atraso no desenvolvimento, sugerindo sua maior vulnerabilidade.
Ainda, estudos sugerem que bebês de mães adolescentes podem apresentar não só atraso em seu desenvolvimento, mas também, problemas psicológicos 4 , deficiência no crescimento3,23, maior morbidade e mortalidade infantil 5,9 quando comparados a outras crianças nascidas de mães adultas. Embora esses dados não tenham sido investigados, o atraso motor em parte dos bebês de mães adolescentes projeta preocupações quanto a possíveis efeitos cumulativos com o passar do tempo.

A falta de cuidados adequados da mãe adolescente já foi observada nos casos em que alterações no desenvolvimento dos bebês foram detectadas 4 . Ao abordar a maternidade adolescente, torna-se necessário enfatizar a problematização do vínculo mãe-bebê, pois este é de grande importância nos primeiros anos de vida da criança, uma vez que, se a ligação entre mãe e filho não for desenvolvida, problemas emocionais 4 e motores 4,24 podem ser potencializados. Estudos reforçam a idéia de que o desenvolvimento adequado depende da qualidade das experiências vividas pela criança e dos vínculos por ela criados; é a mãe que fornece as matrizes organizadoras ao bebê, estimulando suas aquisições motoras desde o nascimento 25 ; se a mãe não estimular adequadamente o bebê, todo o processo fica comprometido 25 .

Vieira et al.3, no entanto, chegaram a resultados opostos a essa idéia e aos do presente estudo, constatando semeIhança no desenvolvimento de crianças de mães adolescentes (em média 17 anos) e adultas, sugerindo cuidados similares nas diferentes faixas etárias. Cabe ressaltar que alguns autores 9,14 chamam a atenção para diferenças entre a maternidade na adolescência precoce e tardia, sugerindo que adolescentes com idade inferior a 17 anos oferecem maior probabilidade de riscos para atraso desenvolvimental no bebê, por estarem expostas a um maior número de fatores restritivos, sendo que as gestantes com 18 ou 19 anos normalmente apresentam características semelhantes às adultas 9,14 . Portanto, deve-se considerar que, ao estudar a maternidade no final da adolescência, os resultados podem ser pouco diferentes dos da maternidade adulta. No presente estudo, porém, não foi controlada a idade das adolescentes à época do parto, impedindo considerações sobre adolescência precoce ou tardia.

Além disso, é preciso considerar que diversos fatores socioculturais interferem nos comportamentos das adolescentes 26 . Trata-se de uma etapa de conflitos psicossociais, o que, somado à imaturidade e baixa auto-estima7,24, poderia resultar em desvantagens para o desenvolvimento da criança. Algumas pesquisas apóiam-se no entendimento de que a gestação na adolescência está cercada de insegurança, tendo sido detectados, como fatores associados, problemas relacionados à baixa escolaridade6,7,10,27, baixo nível de instrução6, baixa renda10,29 e pouca profissionalização dos pais 6,7 , fatores esses sem relação direta com a adolescência. Mais provavelmente freqüentes nessa idade seriam a situação conjugal instável7,29 e apoio familiar ausente ou insuficiente 8 , o que tornaria essa fase inapropriada para a maternidade, aumentando a vulnerabilidade da adolescente no que se refere aos cuidados com a própria saúde e com a do bebê. Entretanto, alguns estudos observaram a inexistência de diferenças entre mães adolescentes e adultas pela procura de serviços de saú$\mathrm{de}^{7}$, ressaltando que as adolescentes são tão cuidadosas com seus bebês quanto as mães de maior idade28,29.

No presente estudo, à parte o achado de diferença no desempenho motor de crianças nascidas de mães adolescentes e mães adultas, não foi encontrada associação entre desempenho motor e a maioria das características biológicas dos bebês analisadas. Esse resultado era esperado, já que foram excluídas da amostra crianças com fatores biológicos associados, tentando pesquisar, em específico, a idade materna como risco para atrasos. No entanto, parece que não seria a idade da mãe um fator isolado causador dessa vulnerabilidade do bebê a atrasos nas aquisições motoras, mas um conjunto de fatores de risco socioculturais $6,9,10,13$.

Assim, dentre as limitações deste estudo, destaca-se o fato de não terem sido pesquisados outros fatores intervenientes, como a idade à época do parto ou nível de escolaridade, nem tampouco características ambientais ou contextuais, que devem ser contempladas em estudos futuros. 
De toda maneira, reitera-se a importância da identificação precoce de crianças expostas a fatores de risco para atraso, possibilitando assim, planejar adequada intervenção, a fim de que as crianças com diagnóstico de atraso possam seguir a mesma seqüência de desenvolvimento que crianças normais 2,30. Os programas de intervenção junto às famílias de bebês de risco têm se mostrado eficazes em ensinar as mães a observar e interpretar os comportamentos dos filhos, tornando-se mais capazes de modificar suas ações de acordo com as necessidades de desenvolvimento do bebê15,16.

Nos Serviços de Saúde Coletiva, avanços vêm sendo logrados pela implementação de estratégias para identificação, captação e acompanhamento de crianças em risco, minimizando e/ou revertendo os efeitos decorrentes destes ${ }^{14}$. Fica evidente a necessidade de medidas preventivas e de apoio, incentivando a integração de profissionais de diferentes áreas, buscando contemplar todos os aspectos que envolvem a saúde e qualidade de vida das adolescentes.

\section{CONCLUSÃO}

$\mathrm{Na}$ busca de associações entre características biológicas dos bebês e seu posterior desenvolvimento motor, foi observada forte associação apenas com o peso ao nascer. Crianças nascidas de adolescentes apresentaram maior porcentagem de atraso no desenvolvimento motor do que as de mães adultas, sugerindo que a idade materna pode representar um risco para atraso no desenvolvimento motor de bebês.

\section{REFERENCIAS}

1 Valentini NC. Percepção de competência e desenvolvimento motor de meninos e meninas: um estudo transversal. Movimento. 2002;8(2):51-62.

2 Halpern R, Giuglian ERJ, Victora CG. Fatores de risco para suspeita de atraso no desenvolvimento neuropsicomotor aos 12 meses de vida. J Pediatr. 2000;76(6):421-8.

3 Vieira MLF, Bicalho GG, Silva JLCP, Barros AAF. Crescimento e desenvolvimento de filhos de mães adolescentes no primeiro ano de vida. Rev Paul Pediatr. 2007;25(4):343-8.

4 Barros MCM, Guinsburg R, Mitsuhiro S, Chalem E, Laranjeira R. Neurocomportamento de recém-nascidos a termo, pequenos para a idade gestacional, filhos de mães adolescentes. J Pediatr. 2008;84(3):217-23.

5 Magalhães ML, Furtado FM, Nogueira MB, Carvalho FH, Almeida FML, Mattar R, et al. Gestação na adolescência precoce e tardia: Há diferença nos riscos obstétricos? Rev Bras Ginecol Obstet. 2006;28:446-52.

6 Gama SGN, Leal MC. Experiência de gravidez na adolescência, fatores associados e resultados perinatais entre puérperas de baixa renda. Cad Saude Publica. 2002;18(1):153-61.

7 Chalem E, Mitsuhiro SS, Ferri CP, Barros MC, Guinsburg R, Laranjeira R. Gravidez na adolescência: perfil sociodemográfico e comportamental de uma população da periferia de São Paulo, Brasil. Cad Saude Publica. 2007;23:177-86.

8 Gama SGN, Szwarcwald CL, Sabroza AR, Branco VC, Leal MC. Fatores associados à assistência pré-natal precária em uma amostra de puérperas adolescentes em maternidades do município do Rio de Janeiro, 19992000. Cad Saude Publica. 2004;20(1):101-11.

9 Simões VMF, Silva AAM, Bettiol H, Lamy-Filho F, Tonial SR, Mochel EG. Características da gravidez na adolescência em São Luís, Maranhão. Rev Saude Publica. 2003;37(5):559-65.
10 Costa COM, Santos CAST, Sobrinho CN, Moura MS, Souza KEPS, Assis DR. Gravidez na adolescência: associação de variáveis sociodemográficas e biomédicas maternas com resultado neonatal; Feira de Santana, Bahia. Rev Bahiana Saude. 2005;29(2):300-12.

11 Gama SGN, Szwarcwald CL, Leal MC, Theme Filha MM. Gravidez na adolescência como fator de risco para baixo peso ao nascer no Município do Rio de Janeiro, 1996 a 1998. Rev Saude Publica. 2001;35(1):74-80.

12 Eickmann SH, Lira PIC, Lima MC. Desenvolvimento mental e motor aos 24 meses de crianças nascidas a termo com baixo peso. Arq Neuropsiquiatr. 2002;60(3):748-54.

13 Costa MC, Santos CA, Sobrinho CLN, Freitas JO, Ferreira KASL, Silva MA, et al. Estudo de partos e nascidos vivos de mães adolescentes e adultas jovens no município de Feira de Santana, Bahia, Brasil, 1998. Cad Saude Publica. 2002;18(3):715-22.

14 Goulart LM, Xavier CC, Goulart EM, Somarriba MG, Almeida CG, Costa AL. Avaliação da ação de vigilância à morbimortalidade infantil na periferia de Belo Horizonte, Minas Gerais. Rev Bras Saude Mater Infant. 2003;3:291-304.

15 Silva OPV. A importância da família no desenvolvimento do bebê prematuro. Psicol Teor Prat. 2002;4:15-24.

16 Silva PL, Santos DCC, Gonçalves VMG. Influência de práticas maternas no desenvolvimento motor de lactentes do 6o ao 120 meses de vida. Rev Bras Fisioter. 2006;10(2):225-31.

17 Abbott AL, Bartlett DJ. Infant motor development and equipment use in the home. Child Care Health Dev. 2001;27(3):295-306.

18 Dunst C, Bruder MB. Valued outcomes of service coordination, early intervention and natural environments. Except Child. 2002;68(3):361-75. 


\section{Referências (cont.)}

19 Willrich A, Azevedo CCF, Fernandes JO. Desenvolvimento motor na infância: influência dos fatores de risco e programas de intervenção. Rev Neurocienc. 2009;17(1):51-6.

20 Thomas RJ, Nelson KJ. Métodos de pesquisa em atividade física. 3a ed. Porto Alegre: Artmed; 2002.

21 Piper MC, Pinnel LE, Darrah J, Maguire T, Byrne PJ. Construction and validation of the Alberta Infant Motor Scale (AIMS). Can J Public Health. 1992;83(Suppl 2):S46-50.

22 Callegari-Jaques S. Bioestatística: princípios e aplicações. Porto Alegre: Artmed; 2003.

23 Esperidião S, Júlio AG, Marcolim M, Silva NA, Oliverio IA, Nascimento JP, et.al. Avaliação dos recém-nascidos de mães adolescentes no Hospital Municipal de Santo André: correlação com algumas variáveis maternas. Pediatr Mod. 1992;28:526-9.

24 Motta MG, Lucion AB, Manfro GG. Efeitos da depressão materna no desenvolvimento neurobiológico e psicológico da criança. Rev Psiquiatr Rio Gd Sul. 2005;27(2):165-76.
25 Gallahue DL, Ozmun JC. Compreendendo o desenvolvimento motor: bebês, crianças, adolescentes e adultos. 3a ed. São Paulo: Phorte; 2005.

26 Feijó RB, Oliveira EA. Comportamento de risco na adolescência. J Pediatr. 2001;77(2):125-34.

27 Dias AB, Aquino EM. Maternidade e paternidade na adolescência: algumas constatações em três cidades do Brasil. Cad Saude Publica. 2006;22:1447-58.

28 Vieira MLF, Silva JL, Barros FAA. A amamentação e a alimentação complementar de filhos de mães adolescentes são diferentes das de filhos de mães adultas? J Pediatr. 2003;79:317-24.

29 Frota DAL, Marcopito LF. Amamentação entre mães adolescentes e não-adolescentes, Monte Claros, MG. Rev Saude Publica. 2004;38:85-92.

30 Ribeiro KSQ. A atuação da fisioterapia na atenção primária à saúde. FisioBrasil. 2002;3(5):311-8. 